\title{
Consumo de cannabis y alteraciones cognitivas en esquizofrenia y primeros episodios psicóticos
}

\section{Cannabis use and cognitive impairment in schizophrenia and first-episode psychosis}

\author{
Leticia García Állvarez *******,****,*****; Jesus J. Gomar***, ******, *******; \\ Ma Paz García-Portilla*,**,***,*****,********; Julio Bobes*,**,***,*****,******** \\ * Instituto de Investigación Sanitaria del Principado de Asturias (ISPA), España \\ ** Área de Psiquiatría. Universidad de Oviedo, España \\ *** Centro de Investigación Biomédica en Red de Salud Mental (CIBERSAM), España \\ **** Instituto de Neurociencias del Principado de Asturias (INEUROPA), España \\ ***** Departamento de Psicología. Universidad de Oviedo, España \\ ****** The Litwin-Zucker Research Center, The Feinstein Institute for Medical Research, Nueva York, NY, Estados Unidos \\ ******* FIDMAG Hermanas Hospitalarias Research Foundation \& CIBERSAM, Sant Boi de Llobregat, España \\ ********* Servicio de Salud del Principado de Asturias. SESPA, España
}

$\mathrm{E}$ 1 uso de cannabis es altamente prevalente a nivel mundial, pero sus efectos sobre el cerebro y la cognición son todavía controvertidos (Block et al., 2000; Jager et al., 2007; Martin-Santos et al., 2010). El uso de esta sustancia se ha relacionado con un mayor riesgo para la psicosis (Casajuana Kögel, López-Pelayo, Balcells-Olivero, Colom y Gual, 2018; Gage, Hickman, y Zammit, 2016; Koskinen, Lohonen, Koponen, Isohanni, y Miettunen, 2010; López Pelayo, de Miquel Montagut, Casajuana Kögel y Balcells Oliveró, 2018; van Os et al., 2002) y, al mismo tiempo, se ha observado cómo el uso de cannabis es más frecuente en la esquizofrenia que en la población general (Barnes, Mutsatsa, Hutton, Watt, y Joyce, 2006). En la población general se estima una tasa de prevalencia de trastornos por consumo de cannabis inferior al 10\% (Moore et al., 2007), mientras que pacientes con esquizofrenia esta tasa ascendería al 27.1\% (Koskinen et al., 2010). Además, si hablamos de consumo de cannabis en primeros episodios psicóticos (PEPs) estas cifras son todavía mayores, reportándose tasas hasta del 65.7\% (Schimmelmann et al., 2012).

Por un lado, las alteraciones cognitivas y las alteraciones en el funcionamiento secundarias a esos déficits se desarrollan de manera temprana en la esquizofrenia y son estables y persistentes a lo largo de toda la evolución del trastorno (Heaton et al., 2001). Por otro, el consumo de cannabis premórbido se ha asociado con más síntomas y un peor funcionamiento en pacientes con trastornos del espectro de la esquizofrenia (Ringen et al., 2016). Sin embargo, los estudios previos sobre el impacto del uso de cannabis a nivel cognitivo en pacientes con esquizofrenia son variados (Potvin, Stavro, y Pelletier, 2012).

\section{Impacto del consumo de cannabis}

Diferentes estudios han observado una asociación positiva entre consumo de cannabis (historia de consumo o consumo actual) y cognición, tanto en pacientes con esquizofrenia (DeRosse, Kaplan, Burdick, Lencz, y Malhotra, 2010; Helle, Loberg, Gjestad, Schnakenberg Martin, y Lysaker, 2017; Meijer et al., 2012; Yucel et al., 2012) como en primeros episodios psicóticos (Cunha et al., 2013; de la Serna et al., 2010; Leeson, Harrison, Ron, Barnes, y Joyce, 2012; Rodriguez-Sanchez et al., 2010; Yucel et al., 2012), aunque a veces este mejor desempeño no se ha encontrado en todas las áreas evaluadas (Bahorik et al., 2014; Schnell, Koethe, Daumann, y Gouzoulis-Mayfrank, 2009). Además, muchos de estos resultados positivos provienen de estudios que incluyen pacientes con historia de consumo a lo largo de la vida más que por consumidores actuales o recientes (Yucel et al., 2012). Sin embargo, otros estudios han observado una relación entre una historia de consu- 
mo de cannabis o consumo actual y un peor rendimiento cognitivo en pacientes con psicosis (Waterreus, Badcock, Di Prinzio, Martin-Iverson, y Morgan, 2017), esquizofrenia (Meijer et al., 2012; Sanchez-Torres et al., 2013) y PEPs(Gonzalez-Pinto et al., 2016), aunque, en estos últimos, ese peor rendimiento se ha relacionado con la gravedad del consumo durante el año previo (Nunez et al., 2016) o con una ausencia de historia familiar de psicosis (Gonzalez-Pinto et al., 2016). Asimismo, se ha observado cómo pacientes con esquizofrenia y PEPs dependientes de cannabis mejoran las funciones cognitivas una vez que cesan el consumo (Rabin et al., 2017; Setien-Suero et al., 2018), siendo esas mejorías menores en controles sanos (Rabin et al., 2017). En otros casos, las diferencias observadas desaparecen al controlar ciertas variables confusoras, como la edad, edad de inicio de la enfermedad, CI premórbido o el índice socioeconómico (Leeson et al., 2012; Power et al., 2015). Finalmente, un menor número de estudios no han observado diferencias cognitivas en función del consumo de cannabis en este tipo de pacientes (Bugra et al., 2013).

Además de todo lo anterior, el mejor o peor desempeño cognitivo en consumidores de cannabis se ha relacionado con el tipo de cuadro diagnóstico, así psicosis más puras con menor interferencia de psicopatología afectiva parecen tener un mejor rendimiento cognitivo (Hanna et al., 2016) o por lo menos no peor (Waterreus et al., 2017) que aquellos otros con otro tipo de psicosis en los que el componente afectivo es fundamental. Asimismo, no sólo diferentes perfiles de pacientes, sino también diferentes perfiles de consumidores de cannabis podrían influir en el rendimiento cognitivo (Schnakenberg Martin et al., 2016).

\section{Variables cognitivas evaluadas}

Los fallos neurocognitivos en pacientes con esquizofrenia han sido ampliamente documentados y se consideran una característica central de la misma (Elvevag y Goldberg, 2000; Green, Kern, y Heaton, 2004), parecen estar presentes desde el primer episodio psicótico e incluso en familiares de primer grado de pacientes con esquizofrenia sin evidencia de síntomas psicóticos (Asarnow et al., 2002) sugiriendo que ciertas alteraciones cognitivas podrían ser componentes de una vulnerabilidad genética para la esquizofrenia. La iniciativa Measurement and Treatment Research to Improve Cognition in Schizophrenia (MATRICS) del National Institute of Mental Health (NIMH) incluyó siete dominios cognitivos como característicos de la esquizofrenia: velocidad de procesamiento, atención/vigilancia, memoria de trabajo, aprendizaje verbal, aprendizaje visual, razonamiento y solución de problemas, y, cognición social (Kern et al., 2008; Nuechterlein et al., 2008).

En estudios llevados a cabo sobre cannabis y cognición en esquizofrenia los diferentes trabajos han evaluado funciones neurocognitivas como la atención/vigilancia, me- moria, aprendizaje verbal, velocidad de procesamiento, funciones ejecutivas, fluidez verbal etc., pero también otras como la cognición social, reconocimiento emocional o teoría de la mente. Sin embargo, las pruebas empleadas para las valoración de las diferentes variables cognitivas han sido diversas y muy pocos estudios han empleado la MATRICS (Bahorik et al., 2014), tal y como recomienda el NIMH.

\section{Variables neurocognitivas}

En cuanto a la capacidad atencional en pacientes con esquizofrenia que consumen cannabis los resultados son controvertidos, unos estudios han observado mayor capacidad atencional en consumidores con un consumo más frecuente (Schnell et al., 2009), aunque otros observan cómo aquellos pacientes consumidores de cannabis que no consumieron en los últimos 30 días tenían mejor rendimiento (Bahorik et al., 2014). Asimismo, un menor CI en consumidores de cannabis predice un peor desempeño atencional en controles sanos, pero no en pacientes con esquizofrenia ni hermanos de estos (Sanchez-Torres et al., 2013). En PEPs, los pacientes con historia de consumo de cannabis o aquellos que habían consumido cannabis antes del inicio de la enfermedad frente aquellos sin historia de consumo de cannabis tenían menos alteraciones atencionales (Cunha et al., 2013; Rodriguez-Sanchez et al., 2010), sin embargo, también se ha observado cómo consumidores graves durante el año previo a la evaluación mostraban un peor desempeño cognitivo frente a los no consumidores o consumidores con un patrón de consumo menos grave (Nunez et al., 2016).

Las tipos de memoria evaluados en los diferentes estudios han sido diversos, memoria visual (Sanchez-Torres et al., 2013; Yucel et al., 2012), verbal (Rabin et al., 2017; Sanchez-Torres et al., 2013; Schnell et al., 2009; Setien-Suero et al., 2018), episódica (Mallet, Ramoz, Le Strat, Gorwood, y Dubertret, 2017), inmediata (Nunez et al., 2016), de trabajo (Gonzalez-Pinto et al., 2016; Meijer et al., 2012; Menendez-Miranda et al., 2019; Nunez et al., 2016; Schnell et al., 2009; Yucel et al., 2012) o a largo plazo (Nunez et al., 2016). Asimismo, los datos de los mismos no son concluyentes. En pacientes con esquizofrenia con historia de consumo de cannabis en algunos casos se ha observado una mejor adquisición de conocimiento (Meijer et al., 2012), mejor desempeño en tareas de memoria (DeRosse et al., 2010; Menendez-Miranda et al., 2019), incluso pacientes que habían sido consumidores graves antes del inicio de la enfermedad parecían mostrar una mejor memoria episódica (Mallet et al., 2017). Sin embargo, en hermanos de pacientes con esquizofrenia una historia de consumo de cannabis a lo largo de la vida se ha asociado con un efecto negativo en la memoria declarativa, tanto visual como verbal (Sanchez-Torres et al., 2013). Por otro lado, en algunos estudios el consumo actual de cannabis en pacientes con 
esquizofrenia se ha asociado con un peor desempeño en memoria de trabajo (Meijer et al., 2012), mientras que en otros un mayor consumo se ha relacionado con un mejor rendimiento (Schnell et al., 2009). Otros encuentran una mejoría en la memoria verbal al dejar el consumo de cannabis en pacientes con esquizofrenia, pero no en controles sanos (Rabin et al., 2017). En PEPs tanto con historia de consumo de cannabis como sin ella se han observado peores puntuaciones en memoria que en los controles sanos (de la Serna et al., 2010). También, se ha observado cómo PEPs consumidores graves de cannabis durante el año previo mostraban alteración en memoria verbal inmediata, a corto plazo y largo plazo, comparados con los no consumidores (Nunez et al., 2016), aunque un estudio observa esta relación entre consumo de cannabis y peor memoria verbal solo en PEPs que no tenían historia familiar de psicosis, pero no en los que sí la tenían. Asimismo, se ha visto cómo los PEPs que dejan el consumo de cannabis muestran una mejoría en memoria verbal (Setien-Suero et al., 2018). En cuanto a memoria de trabajo, los PEPs con consumo de cannabis parecen tener un peor rendimiento (Gonzalez-Pinto et al., 2016).

Con respecto al aprendizaje verbal en pacientes con esquizofrenia, de nuevo, resultados contradictorios. Se ha observado por un lado, cómo pacientes con esquizofrenia con historia de consumo de cannabis se desempeñan mejor en aprendizaje verbal frente a pacientes sin historia de consumo (DeRosse et al., 2010), pero al mismo tiempo el consumo actual de cannabis se asocia a un peor desempeño en aprendizaje verbal inmediato (Meijer et al., 2012). En PEPs tanto pacientes con historia de consumo de cannabis como sin ella, obtienen peores puntuaciones en aprendizaje verbal que los controles sanos (de la Serna et al., 2010).

El consumo de cannabis a lo largo de la vida en personas con trastornos psicóticos (Power et al., 2015) o esquizofrenia (Menendez-Miranda et al., 2019) también se ha asociado con la velocidad de procesamiento, pero de nuevo se observan resultados dispares, mientras que unos observan un mejor desempeño (DeRosse et al., 2010; Rabin, Zakzanis, Daskalakis, y George, 2013), otros encuentran un peor rendimiento (Meijer et al., 2012). Sin embargo, una vez que se controlan ciertas variables confusoras (edad, edad de inicio de la enfermedad, CI premórbido y el índice socioeconómico) desaparece la asociación entre cannabis y velocidad de procesamiento (Power et al., 2015). Asimismo, en hermanos de pacientes con esquizofrenia una historia de consumo de cannabis a lo largo de la vida parece tener un efecto negativo en velocidad de procesamiento, pero esto no ocurre en controles sanos, a no ser que se incluya el uso de tabaco, es decir, se observa una relación negativa entre el uso de cannabis a lo largo de la vida y tabaco y la velocidad de procesamiento en controles sanos (Sanchez-Torres et al., 2013). En PEPs el consumo grave de cannabis grave durante el año previo se asocia a una menor velocidad de procesamiento (Nunez et al., 2016).

Finalmente, las funciones ejecutivas se han asociado de manera positiva con el uso de cannabis en pacientes con esquizofrenia (Helle et al., 2017; Schnell et al., 2009). En PEPs una historia de consumo de cannabis antes del inicio de la enfermedad se ha asociado con un mayor rendimiento en funciones ejecutivas (Cunha et al., 2013; Rodriguez-Sanchez et al., 2010; Yucel et al., 2012). Sin embargo, una variable que parece mediar de nuevo son los antecedentes familiares de psicosis, ya que PEPs con consumo de cannabis sin historia familiar de psicosis se desempeñan peor en funciones ejecutivas, mientras que los que tenían historia familiar de psicosis tuvieron un mejor rendimiento (Gonzalez-Pinto et al., 2016).

\section{Cognición social}

Con respecto a la relación entre consumo de cannabis y cognición social en pacientes con esquizofrenia los datos, de nuevo, son contradictorios. Mientas que unos observan un mejor reconocimiento facial de emociones y de la identidad en pacientes con esquizofrenia con historia de consumo de cannabis (Meijer et al., 2012) otros observan un peor reconocimiento emocional (Helle et al., 2017) y cognición social (Sanchez-Torres et al., 2013) y un mejor rendimiento cuando se cesa el consumo de cannabis en los 30 días previos a la evaluación. Por otro lado, otros no observan relación entre este consumo y la teoría de la mente (Helle et al., 2017).

\section{Posibles explicaciones de las inconsistencias en los resultados}

Se han planteado diferentes hipótesis para explicar los resultados observados. Los pacientes consumidores de cannabis podrían constituir un subgrupo de pacientes con mejor ajuste premórbido y mejores funciones cognitivas prefrontales premórbidas (Rodriguez-Sanchez et al., 2010). Quizá estemos hablando de pacientes cuyo proceso etiológico del cuadro psicótico es diferente, siendo los PEPs que consumen cannabis y desarrollan psicosis un grupo de pacientes que tienen un menor daño a nivel del neurodesarrollo, y, por tanto, una mayor reserva cognitiva que otros pacientes psicóticos (Cunha et al., 2013). El uso de cannabis podría precipitar en inicio de la psicosis en personas que de otra forma podrían tener un buen pronóstico con un inicio más tardío o incluso sin que llegasen a desarrollar el cuadro, debido a la acción tóxica del cannabis más que por una enfermedad intrínsecamente más grave (Leeson et al., 2012). Por otro lado, se ha planteado cómo una mejor función cognitiva en pacientes con esquizofrenia con consumo de cannabis puede reflejar una menor vulnerabilidad para la psicosis (un mayor nivel de funcionamiento y 
habilidad cognitiva) comparados con otros pacientes con esquizofrenia (Schnell et al., 2009). También se ha intentado explicar el hecho de que los pacientes con esquizofrenia con historia de consumo de cannabis tengan un mejor desempeño cognitivo aludiendo a una mayor cognición social, pero los resultados no apoyan esa hipótesis (Helle et al., 2017). Asimismo, se ha planteado cómo el diferente patrón de asociaciones entre el uso de cannabis y el desempeño cognitivo en pacientes con esquizofrenia comparados con hermanos de pacientes o controles sanos podría explicarse por el impacto negativo que ya produce de por sí la enfermedad (Sanchez-Torres et al., 2013). Finalmente, parece que la dosis de cannabis consumida puede ser una variable que influye en las diferencias encontradas a nivel cognitivo (Nunez et al., 2016).

\section{Conclusiones}

Los diferentes estudios llevados a cabo sobre psicosis, cannabis y cognición difieren en aspectos que quizá puedan ser relevantes y tengan que ver con las diferencias observadas en los resultados. Así, hay estudios que se han centrado en la psicosis en general, incluyendo psicosis afectivas, frente a otros que se ha centrado específicamente en la esquizofrenia o en primeros episodios psicóticos. Sin embargo, bien es sabido que estamos ante cuadros con características diferenciales cuyo estudio debe realizarse de manera aislada, para poder contrastar y replicar los resultados. De igual forma, algunos estudios observan diferencias en función de si esos pacientes tienen antecedentes familiares de psicosis o no, por tanto, es otra variable que habría que tener en consideración a la hora de replicar los diferentes resultados. Asimismo, cuando hablamos de cannabis, hay estudios que se han centrado en una historia de consumo de cannabis a lo largo de la vida, pudiendo haber sido ese consumo relativamente puntual o haber pasado años desde el último, frente a otros que se centran en un consumo actual, cuando el mismo también puede tener patrones diferentes (leve, moderado, grave) con consecuencias también diferentes. De igual modo, es importante considerar ese consumo con respecto al momento de inicio de la enferme$\mathrm{dad}$, si fue antes, si se mantuvo durante los primeros años o si se produjo de manera ininterrumpida. Finalmente, cuando hablamos de alteraciones cognitivas, las evaluaciones han sido dispares, no sólo en las variables cognitivas evaluadas o en el número de áreas incluidas, sino también en las pruebas empleadas. Existe un consenso en la evaluación de la cognición en pacientes con esquizofrenia a través de la MATRICS, sin embargo hay pocos estudios que utilicen esta prueba. Por todo ello, antes de poder llegar a conclusiones firmes sobre los resultados obtenidos es necesario que haya uniformidad en el tipo de pacientes incluidos, así como en las variables evaluadas y en la forma de evaluar las mismas. Al mismo tiempo sería muy importante realizar estudios longitudinales para ver los cambios en la variables cognitivas en función del patrón de consumo que se tiene en esos momentos, o que se ha tenido, e incluir todas aquellas variables confusoras que puedan estar interviniendo.

\section{Agradecimientos}

Los autores quieren agradecer a la Fundación para la Investigación e Innovación Biosanitaria del Principado de Asturias (Finba) el apoyo económico.

\section{Conflicto de intereses}

Los autores declaran que no existe conflicto de interés.

\section{Referencias}

Asarnow, R. F., Nuechterlein, K. H., Subotnik, K. L., Fogelson, D. L., Torquato, R. D., Payne, D. L., . . Guthrie, D. (2002). Neurocognitive impairments in nonpsychotic parents of children with schizophrenia and attentiondeficit/ hyperactivity disorder: the University of California, Los Angeles Family Study. Archives of General Psychiatry, 59, 1053-1060. doi:10.1001/archpsyc.59.11.1053.

Bahorik, A. L., Cornelius, J. R., Bangalore, S. S., Newhill, C. E., Keshavan, M. S. y Eack, S. M. (2014). Brief Report: The Impact of Alcohol and Cannabis Misuse on Cognition among Individuals with Schizophrenia. Schizophrenia Research: Cognition, 1, 160-163. doi:10.1016/j. scog.2014.08.001.

Barnes, T. R., Mutsatsa, S. H., Hutton, S. B., Watt, H. C. y Joyce, E. M. (2006). Comorbid substance use and age at onset of schizophrenia. British Journal of Psychiatry, 188, 237-242. doi:10.1192/bjp.bp.104.007237.

Block, R. I., O’Leary, D. S., Ehrhardt, J. C., Augustinack, J. C., Ghoneim, M. M., Arndt, S. y Hall, J. A. (2000). Effects of frequent marijuana use on brain tissue volume and composition. Neuroreport, 11, 491-496. doi:10.1097/00001756-200002280-00013.

Bugra, H., Studerus, E., Rapp, C., Tamagni, C., Aston, J., Borgwardt, S. y Riecher-Rossler, A. (2013). Cannabis use and cognitive functions in at-risk mental state and first episode psychosis. Psychopharmacology (Berl), 230, 299308. doi:10.1007/s00213-013-3157-y.

Casajuana Kögel, C., López-Pelayo, H., Balcells-Olivero, M.M., Colom. J. y Gual A. (2018). Psychoactive constituents of cannabis and their clinical implications: a systematic review. Adicciones, 30, 140-151. doi:10.20882/ adicciones.858.

Cunha, P. J., Rosa, P. G., Ayres Ade, M., Duran, F. L., Santos, L. C., Scazufca, M., . . . Schaufelberger, M. S. (2013). Cannabis use, cognition and brain structure in first-episode psychosis. Schizophrenia Research, 147, 209-215. doi:10.1016/j.schres.2013.04.009. 
de la Serna, E., Mayoral, M., Baeza, I., Arango, C., Andres, P., Bombin, I., . . . Castro-Fornieles, J. (2010). Cognitive functioning in children and adolescents in their first episode of psychosis: differences between previous cannabis users and nonusers. The Journal of Nervous and Mental Disease, 198, 159-162. doi:10.1097/NMD. 0b013e3181cc0d41.

DeRosse, P., Kaplan, A., Burdick, K. E., Lencz, T. y Malhotra, A. K. (2010). Cannabis use disorders in schizophrenia: effects on cognition and symptoms. Schizophrenia Research, 120, 95-100. doi:10.1016/j.schres.2010.04.007.

Elvevag, B. y Goldberg, T. E. (2000). Cognitive impairment in schizophrenia is the core of the disorder. Critical Reviews ${ }^{T M}$ in Neurobiology, 14, 1-21. doi:10.1615/CritRevNeurobiol.v14.i1.10.

Gage, S.H., Hickman, M. y Zammit, S. (2016). Association Between Cannabis and Psychosis: Epidemiologic Evidence. Biological Psychiatry, 79, 549-556. doi:10.1016/j. biopsych.2015.08.001.

Gonzalez-Pinto, A., Gonzalez-Ortega, I., Alberich, S., Ruiz de Azua, S., Bernardo, M., Bioque, M., . . . PEPs Group. (2016). Opposite Cannabis-Cognition Associations in Psychotic Patients Depending on Family History. Public Library of Science One, 11, e0160949. doi:10.1371/journal.pone.0160949.

Green, M. F., Kern, R. S. y Heaton, R. K. (2004). Longitudinal studies of cognition and functional outcome in schizophrenia: implications for MATRICS. Schizophrenia Research, 72, 41-51. doi:10.1016/j.schres.2004.09.009.

Hanna, R. C., Shalvoy, A., Cullum, C. M., Ivleva, E. I., Keshavan, M., Pearlson, G., . . G Ghose, S. (2016). Cognitive Function in Individuals With Psychosis: Moderation by Adolescent Cannabis Use. Schizophrenia Bulletin, 42, 1496-1503. doi:10.1093/schbul/sbw030.

Heaton, R. K., Gladsjo, J. A., Palmer, B. W., Kuck, J., Marcotte, T. D. y Jeste, D. V. (2001). Stability and course of neuropsychological deficits in schizophrenia. Archives of General Psychiatry, 58, 24-32. doi:10.1001/archpsyc.58.1.24.

Helle, S., Loberg, E. M., Gjestad, R., Schnakenberg Martin, A. M. y Lysaker, P. H. (2017). The positive link between executive function and lifetime cannabis use in schizophrenia is not explained by current levels of superior social cognition. Psychiatry Research, 250, 92-98. doi:10.1016/j.psychres.2017.01.047.

Jager, G., Van Hell, H. H., De Win, M. M., Kahn, R. S., Van Den Brink, W., Van Ree, J. M. y Ramsey, N. F. (2007). Effects of frequent cannabis use on hippocampal activity during an associative memory task. European Neuropsychopharmacology, 17, 289-297. doi:10.1016/j.euroneuro.2006.10.003.

Kern, R. S., Nuechterlein, K. H., Green, M. F., Baade, L. E., Fenton, W. S., Gold, J. M., . . Marder, S. R. (2008). The MATRICS Consensus Cognitive Battery, part 2: co-nor- ming and standardization. The American Journal of Psychiatry, 165, 214-220. doi:10.1176/appi.ajp.2007.07010043.

Koskinen, J., Lohonen, J., Koponen, H., Isohanni, M. y Miettunen, J. (2010). Rate of cannabis use disorders in clinical samples of patients with schizophrenia: a meta-analysis. Schizophrenia Bulletin, 36, 1115-1130. doi:10.1093/ schbul/sbp031.

Leeson, V. C., Harrison, I., Ron, M. A., Barnes, T. R. y Joyce, E. M. (2012). The effect of cannabis use and cognitive reserve on age at onset and psychosis outcomes in first-episode schizophrenia. Schizophrenia Bulletin, 38, 873-880. doi:10.1093/schbul/sbq153.

López Pelayo, H., de Miquel Montagut, L., Casajuana Kögel, C. y Balcells Oliveró, M. (2018). Post-truth Cannabis use: back to evidence-based medicine. Adicciones, 30, 237-242. doi:10.20882/adicciones.

Mallet, J., Ramoz, N., Le Strat, Y., Gorwood, P. y Dubertret, C. (2017). Heavy cannabis use prior psychosis in schizophrenia: clinical, cognitive and neurological evidences for a new endophenotype? European Archives of Psychiatry and Clinical Neuroscience, 267, 629-638. doi:10.1007/s00406-017-0767-0.

Martin-Santos, R., Fagundo, A. B., Crippa, J. A., Atakan, Z., Bhattacharyya, S., Allen, P., . . McGuire, P. (2010). Neuroimaging in cannabis use: a systematic review of the literature. Psychological Medicine, 40, 383-398. doi:10.1017/ S0033291709990729.

Meijer, J. H., Dekker, N., Koeter, M. W., Quee, P. J., van Beveren, N. J., Meijer, C. J., . . O Outcome of Psychosis, Investigators. (2012). Cannabis and cognitive performance in psychosis: a cross-sectional study in patients with non-affective psychotic illness and their unaffected siblings. Psychological Medicine, 42, 705-716. doi:10.1017/ S0033291711001656.

Menendez-Miranda, I., García-Alvarez, L., García-Portilla, P., Gonzalez-Blanco, L., Saiz, P. y Bobes, J. (2019). History of lifetime cannabis use is associated with better cognition and worse real-world functioning in schizophrenia spectrum disorders. European Addiction Research, 25, 111-118. doi:10.1159/000497317.

Moore, T. H., Zammit, S., Lingford-Hughes, A., Barnes, T. R., Jones, P. B., Burke, M. y Lewis, G. (2007). Cannabis use and risk of psychotic or affective mental health outcomes: a systematic review. Lancet, 370, 319-328. doi:10.1016/S0140-6736(07)61162-3.

Nuechterlein, K. H., Green, M. F., Kern, R. S., Baade, L. E., Barch, D. M., Cohen, J. D., . . Marder, S. R. (2008). The MATRICS Consensus Cognitive Battery, part 1: test selection, reliability, and validity. The American Journal of Psychiatry, 165, 203-213. doi:10.1176/appi. ajp.2007.07010042.

Nunez, C., Ochoa, S., Huerta-Ramos, E., Banos, I., Barajas, A., Dolz, M., . . . Usall, J. (2016). Cannabis use and cognitive function in first episode psychosis: differential 
effect of heavy use. Psychopharmacology (Berl), 233, 809821. doi:10.1007/s00213-015-4160-2.

Potvin, S., Stavro, K. y Pelletier, J. (2012). Paradoxical cognitive capacities in dual diagnosis schizophrenia: the quest for explanatory factors. Journal of Dual Diagnosis, 8, 35-47. doi:10.1080/15504263.2012.648549.

Power, B. D., Dragovic, M., Badcock, J. C., Morgan, V. A., Castle, D., Jablensky, A. y Stefanis, N. C. (2015). No additive effect of cannabis on cognition in schizophrenia. Schizophrenia Research, 168, 245-251. doi:10.1016/j. schres.2015.06.026.

Rabin, R. A., Barr, M. S., Goodman, M. S., Herman, Y., Zakzanis, K. K., Kish, S. J., . . George, T. P. (2017). Effects of Extended Cannabis Abstinence on Cognitive Outcomes in Cannabis Dependent Patients with Schizophrenia vs Non-Psychiatric Controls. Neuropsychopharmacology, 42, 2259-2271. doi:10.1038/npp.2017.85.

Rabin, R. A., Zakzanis, K. K., Daskalakis, Z. J. y George, T. P. (2013). Effects of cannabis use status on cognitive function, in males with schizophrenia. Psychiatry Research, 206, 158-165. doi:10.1016/j.psychres.2012.11.019.

Ringen, P. A., Nesvag, R., Helle, S., Lagerberg, T. V., Lange, E. H., Loberg, E. M., . . . Melle, I. (2016). Premorbid cannabis use is associated with more symptoms and poorer functioning in schizophrenia spectrum disorder. Psychological Medicine, 46, 3127-3136. doi:10.1017/ S0033291716001999.

Rodriguez-Sanchez, J. M., Ayesa-Arriola, R., Mata, I., Moreno-Calle, T., Perez-Iglesias, R., Gonzalez-Blanch, C., ... Crespo-Facorro, B. (2010). Cannabis use and cognitive functioning in first-episode schizophrenia patients. Schizophrenia Research, 124, 142-151. doi:10.1016/j. schres.2010.08.017.

Sanchez-Torres, A. M., Basterra, V., Rosa, A., Fananas, L., Zarzuela, A., Ibanez, B., . . Cuesta, M. J. (2013). Lifetime cannabis use and cognition in patients with schizophrenia spectrum disorders and their unaffected siblings. European Archives of Psychiatry and Clinical Neuroscience, 263, 643-653. doi:10.1007/s00406-013-0404-5.

Schimmelmann, B.G., Conus, P., Cotton, S., Kupferschmid, S., McGorry, P.D. y Lambert, M. (2012). Prevalence and impact of cannabis use disorders in adolescents with early onset first episode psychosis. European Psychiatry, 27, 463-469. doi:10.1016/j.eurpsy.2011.03.001.

Schnakenberg Martin, A.M., Bonfils, K.A., Davis, B.J., Smith, E.A., Schuder, K. y Lysaker, P.H. (2016). Compared to high and low cannabis use, moderate use is associated with fewer cognitive deficits in psychosis. Schizophrenia Research: Cognition, 6, 15-21. doi:10.1016/j. scog.2016.09.001.

Schnell, T., Koethe, D., Daumann, J. y Gouzoulis-Mayfrank, E. (2009). The role of cannabis in cognitive functioning of patients with schizophrenia. Psychopharmacology (Berl), 205, 45-52. doi:10.1007/s00213-009-1512-9.
Setien-Suero, E., Martinez-Garcia, O., de la Foz, V. O., Vazquez-Bourgon, J., Correa-Ghisays, P., Ferro, A., . . . Ayesa-Arriola, R. (2018). Age of onset of Cannabis use and cognitive function in first-episode non-affective psychosis patients: Outcome at three-year follow-up. Schizophrenia Research, 201, 159-166. doi:10.1016/j. schres.2018.05.036.

van Os, J., Bak, M., Hanssen, M., Bijl, R. V., de Graaf, R. y Verdoux, H. (2002). Cannabis use and psychosis: a longitudinal population-based study. American Journal of Epidemiology, 156, 319-327. doi:10.1093/aje/kwf043.

Waterreus, A., Badcock, J. C., Di Prinzio, P., Martin-Iverson, M. y Morgan, V. A. (2017). The impact of current cannabis use on general cognitive function in people with psychotic illness. Schizophrenia Research, 190, 164-171. doi:10.1016/j.schres.2017.03.038.

Yucel, M., Bora, E., Lubman, D. I., Solowij, N., Brewer, W. J., Cotton, S. M., . . Pantelis, C. (2012). The impact of cannabis use on cognitive functioning in patients with schizophrenia: a meta-analysis of existing findings and new data in a first-episode sample. Schizophrenia Bulletin, 38, 316-330. doi:10.1093/schbul/sbq079. 